weakened by relegating part of the punch line to an appendix, however. A discussion of the bag model is murky and out of date, and raises, but does not settle, the question of the $\eta$ and $\eta^{\prime}$ masses. The chapter on deeply inelastic leptonhadron scattering, the source of much of our experimental information on the structure of the proton, suffers from inconsistent, non-standard notation and ancient data. The essential ideas are here, but in a rather graceless form. Notational to and fro that might seem spontaneous on a blackboard appears simply indecisive in print.

The treatment of electroweak interactions analyses the phenomenology of neutral current interactions and properties of gauge bosons before coming to terms with the spontaneous breaking of the electroweak gauge symmetry. Notwithstanding an insightful discussion of the recognition of the gauge symmetry, this chapter does not entirely succeed. When intuitive arguments and elementary techniques lead without noticeable effort to insights about profound and challenging problems, it is wondrous to behold. But when the approach comes up short, it simply calls attention to the self-imposed handicaps and interferes with the subject itself. Again there is all too frequently an annoying imprecision: a charge asymmetry in the reaction $e^{+} e^{-} \rightarrow \mu^{+} \mu^{-}$is not a parity-violating effect, but only evidence for some mechanism besides singlephoton exchange. The oral tradition must stand for impeccable standards of scholarship, as well as insight. When at last it appears, the treatment of hidden symmetries contains some instructive remarks on the analogy with superconductivity and ferromagnetism, and is worth reading.

Overall, there is a disappointing inattention to detail in the production of the book. Assiduous copyediting should have removed awkward turns of phrase, disagreements between subject and verb, and artefacts from the original manuscript. Typographical errors are common enough to be intrusive.

Despite the authors' contagious love of the subject and their desire to communicate the excitement and progress of recent years to a broad audience, Concepts of Particle Physics is no royal road to wisdom. Viewed not as a textbook to be studied, but as an anthology of lectures to be browsed, it has a place in departmental libraries.

Chris Quigg is Deputy Director for Operations of the Superconducting Super Collider Central Design Group, Lawrence Berkeley Laboratory, One Cyclotron Road, Berkeley, California 94720, USA, and Visiting Professor in the Department of Physics, University of California at Berkeley.

\section{No room for loners}

\author{
Robert M. May \& Naomi E. Pierce
}

Animal Societies: Theories and Facts. Edited by Yosiaki Itô, Jerram L. Brown and Jiro Kikkawa. Japan Scientific Societies Press, 2-10 Hongo, 6-chome, Bunkyo-ku, Tokyo 113, Japan: 1987. Pp. 291. $¥ 8,200$.

From 1983 to 1986, the Japanese Ministry of Education, Science and Culture sponsored a special research project on "Biological Aspects of Optimal Strategy and Social Structure". This project, directed by $\mathrm{Ei}$ Teramoto, built on traditional Japanese strengths in ethology and population biology to fund more than 100 behavioural ecologists. The enterprise was the second largest ever undertaken by Japanese ecologists (the largest being the IBP project in the 1960s), and culminated in an international symposium in Kyoto in July 1986. This book represents the proceedings of the symposium.

Because biologists all over Japan were invited to apply for funding from the project, it is not surprising that a wide range of topics was studied, within the broadly unifying theme of the evolution of social behaviour. The book thus contains a rather eclectic collection of papers, with contributions on wasps, aphids and without any explicit kin recognition.

Animal Societies contains several outstanding examples of the work of younger people who were funded by the project. M. Hiraiwa-Hasegawa's analysis of infanticide in primates draws from comparative studies and from her own research on chimpanzees to give an excellent review of a difficult subject. S. Aoki's discussion of the evolution of sterile soldiers in aphids is a highlight of the book: Aoki first discovered the phenomenon of soldier aphids while he was a graduate student in Hokkaido, and he has gone on to describe about 20 species which show this caste differentiation. In this paper, Aoki has synthesized some of these separate observations into a fascinating comparative study that makes plausible arguments about the evolution of sterile soldiers in the cerataphidine horned aphids.

$H$. Ubukata discusses the behaviour and mating system of the dragonfly Cordulia aenea amurensis, and develops a model to explain the searching behaviour of males (who often patrol in straight lines). Ubukata concludes that at high male densities there is an optimal length of territory, with time being wasted in conflict if longer segments are patrolled; at low male densities, the probability of mating success increases as patrol length increases. These theoretical conclusions agree well with observations. Additional interesting papers were presented by other young Japanese scientists at satellite meetings following the main session, and it is perhaps a pity that some of these were not included in the book.

The overseas visitors to the symposium included M.J. West-Eberhart, A.H. Harcourt, M. Taborsky, J. Kikkawa, J.L. Brown and J.R. Krebs, who also contribute stimulating essays on topics central to the theme of the symposium. Kikkawa's paper is notable because it presents new data on social relationships and fitness from his long-term study of silvereye populations on Heron Island.

In our view, the most engagingly original contribution comes from W.D. Hamilton, who turns a tired subject on its head and muses, not about why animals such as hymenopterans and aphids are social, but about why they are not even more cooperative than they already are: "Why is the number of [aphid] morphs so small, and the degree of cooperation effected so slight, compared to conditions found in the assemblage of cells that make up the metazoan body?". Hamilton concludes on a quirky note, with a futuristic view of the final world organism as a "very selfassured, competent, and doubtless, for this case, female, medical student". For an explanation of this vision, you must see the book.

Robert M. May and Naomi E. Pierce are in the Biology Department, Princeton University, Princeton, New Jersey 08544, USA. 